\title{
An In Vivo Spectral Multiplexing Approach for the Cooperative Imaging of Different Disease-Related Biomarkers with Near-Infrared Fluorescent Förster Resonance Energy Transfer Probes
}

\author{
Corinna Busch ${ }^{1}$, Tom Schröter ${ }^{1}$, Markus Grabolle ${ }^{2}$, Matthias Wenzel ${ }^{3}$, Hanne Kempe ${ }^{1}$, Werner A. Kaiser ${ }^{4}$, \\ Ute Resch-Genger ${ }^{2}$, and Ingrid Hilger ${ }^{1}$ \\ ${ }^{1}$ Institute of Diagnostic and Interventional Radiology, Department of Experimental Radiology, University Hospital Jena, Jena, \\ Germany; ${ }^{2}$ BAM Bundesanstalt für Materialforschung und prüfung, Berlin, Germany; ${ }^{3}$ Dyomics GmbH, Jena, Germany; and \\ ${ }^{4}$ Institute of Diagnostic and Interventional Radiology, University Hospital Jena, Jena, Germany
}

\begin{abstract}
In recent years, much progress has been made in analyzing the molecular origin of many diseases in vivo. For most applications, attention has been devoted to the detection of single molecules only. In this study, we present a proof of concept for the straightforward monitoring of interactions between different molecules via Förster resonance energy transfer (FRET) in an in vivo spectral multiplexing approach using conventional small organic dyes covalently attached to antibodies. Methods: We coupled the fluorophores DY-682 (donor; absorption [abs]/ emission [em], 674/712 nm), DY-505 (control donor; abs/em, 498/529 nm), and DY-782 (acceptor; abs/em, 752/795 nm) to the model antibody lgG. The occurrence of FRET between these fluorophores was assessed in vitro for conjugate mixtures adsorbed onto membranes, after accumulation into the phagocytic compartment of macrophages (J774 cells), and in vivo in a mouse edema model using a whole-body animal imaging system with multispectral analysis features. Results: When the free acceptor DY-782 was combined with the DY-682 donor, FRET occurred as a consequence of small dye-to-dye distances, unlike the case for mixtures of the dyes DY-782 and DY-505. Our proof of concept was also transferred to living cells after internalization of the DY-682-lgG-DY-782-IgG pair into macrophages and finally to animals, where intermolecular FRET was observed after systemic probe application in vivo in edema-bearing mice. Conclusion: Our simple cooperative-imaging approach enables the noninvasive detection of the presence of two or principally even more neighboring disease-related biomarkers. This finding is of high relevance for the in vivo identification of complex biologic processes requiring strong spatial interrelations of target molecules in key pathologic activation processes such as inflammation, cancer, and neurodegenerative diseases.
\end{abstract}

\footnotetext{
Received Jun. 24, 2011; revision accepted Nov. 29, 2011.

For correspondence or reprints contact: Ingrid Hilger, Institute of Diagnostic and Interventional Radiology, University Hospital Jena, Erlanger Allee 101, 07747 Jena, Germany.

E-mail: Ingrid.Hilger@med.uni-jena.de

Published online Mar. 9, 2012.

COPYRIGHT @ 2012 by the Society of Nuclear Medicine, Inc.
}

Key Words: FRET; IgG antibodies; in vivo molecular imaging; near-infrared (NIR) fluorophores; multiplexing; cooperative signaling

J Nucl Med 2012; 53:638-646

DOI: 10.2967/jnumed.111.094391

$\mathbf{U}$ nderstanding the molecular basis and function of diseases is known to be decisive for the identification of new drug targets and the recognition of new strategies for determining the diagnosis and prognosis early. Extensive research in this field has demonstrated the complexity of interactions that take place on the molecular level in the course of disease progression-interactions that involve alterations in the genome, transcription, processing of transcripts, transport, and product degradation, among others. This experience pioneered the development of in vivo imaging techniques at the molecular level to better identify defined disease states. Among them, optical imaging turned out to be a favorable modality, since it does not require ionizing radiation, is inexpensive, and comprises an easy-tooperate instrumentation providing high detection sensitivity and multiplexing capabilities. Different optical approaches have been considered so far. Bioluminescence imaging offers the possibility to study ongoing molecular processes by the use of genetically encoded luminescent proteins incorporated into cells or laboratory animals. However, these luminescent proteins do not enable deep tissue penetration; they are hampered by tissue-related absorption and lead to comparatively low tumor-to-background ratios due to a distinct signal contribution from tissue-related autofluorescence. Additionally, the incorporation of foreign genetic material lowers the clinical potential significantly. In contrast, optical probes with absorption and emission bands in the near-infrared region (650-900 $\mathrm{nm})(1)$ enable a relatively deep tissue penetration due to reduced absorption by 
water and hemoglobin and yield an improved signal-to-noise ratio (2,3). Consequently, the clinical potential of near-infrared fluorescence (NIRF) imaging is considered to be high. So far, most in vivo NIRF optical imaging approaches have been based on the detection of informative biomarker expression target tissues only.

Förster resonance energy transfer (FRET) may offer the potential to image molecular cooperation representing key functional markers in disease, such as defined biochemical activation processes, providing more accurate insights into highly tuned and regulated disease processes. FRET is a phenomenon that takes place when 2 different chromophores (donor and acceptor) with overlapping emission/absorption spectra undergo long-range dipole-dipole coupling $(3,4)$. Several interesting approaches have been reported from microscopic analysis in vitro (5-8) and ultimately also in vivo (9). In the latter case, mutants of green fluorescent protein $(10,11)$ with varying spectral properties have been used together with recombinant techniques to introduce those fused proteins containing the respective FRET donor and acceptor chromophores into cell systems.

With the aim of developing simple approaches toward multiplexed in vivo imaging of biomarker interrelations using a conventional animal imager for multispectral imaging, we describe here an in vivo intermolecular FRET approach that relies on probes in which donor and acceptor are bound to different biomacromolecules. This technique exploits 2 nearinfrared-absorbing and -emitting asymmetric cyanine dyes, each covalently attached to a model biomarker-specific ligand (antibody, IgG) because both probes can be condensed into endolysosomes of monocytes or macrophages via phagocytosis. As a first proof for our envisioned concept, we chose DY682 and DY-782 antibody conjugates, with DY-682 acting as donor and DY-782 as acceptor of this potential FRET pair. The advantage of these near-infrared fluorophores lies in their higher fluorescence quantum yields, enhanced thermal stability, and reduced cytotoxicity in endothelial cells and macrophages compared with indocyanine green (12-15). The close proximity of both probes is expected to result in FRET. The enhancement of the fluorescence intensity of DY782 is indicative of the simultaneous presence of both probes and thus, eventually, of 2 specific molecular structures in close proximity.

\section{MATERIALS AND METHODS}

\section{Synthesis of NIRF Probes}

For preparation of the fluorescent probes, polyclonal IgG from rabbit serum (Sigma-Aldrich $\mathrm{GmbH}$ ) was used. $N$-hydroxysuccinimidyl esters of the fluorescent dyes DY-505, DY-682, and DY-782 were provided by Dyomics $\mathrm{GmbH}$ and coupled to IgG according to the manufacturer's instructions. Briefly, IgG antibodies (1 mg) were dissolved in $100 \mathrm{mM} \mathrm{NaHCO}$ and $500 \mathrm{mM} \mathrm{NaCl}, \mathrm{pH}$ 8.0. The dye, dissolved in dimethyl formamide at a concentration of $20 \mu \mathrm{g} / \mu \mathrm{L}$, was added in a molecular weight ratio of $6: 1$ (dye:IgG). After incubation of the reaction mixture for $2 \mathrm{~h}$ at room temperature, the fluorescent probes were purified chromatographically. The labeling densities (dye-to-protein ratios) were calculated from the absorbance of the conjugate at $280 \mathrm{~nm}$ (corrected for dye contribution) and the integral absorbance at the dye's longest wavelength absorption band $(16,17)$.

\section{Spectroscopic Characterization of Near-Infrared Dyes}

The absorption spectra were recorded on a Cary 5000 ultraviolet/visible light/near-infrared spectrophotometer (Varian Inc.) using 1-cm quartz cells. The fluorescence spectra were measured with a calibrated SLM 8100 spectrofluorometer (Spectronics Instruments) with Glan Thompson polarizers placed in the excitation and emission channels and set to $0^{\circ}$ and $54.7^{\circ}$, respectively (18). For the fluorescence measurements and determination of the fluorescence quantum yields, quartz cuvettes with an optical path length of $1 \mathrm{~cm}$ in the direction of the excitation light and 0.2 $\mathrm{cm}$ in the direction of the fluorescence were used. The fluorescence quantum yields were determined by following a previously described procedure (19) using the quantum yield standards $1,1^{\prime}, 3,3,3^{\prime}, 3^{\prime}$-hexamethylindotricarbocyanine iodide (solvent, ethanol; quantum yield, 0.33) for DY-682 and DY-782 and rhodamine 6G (solvent, ethanol; quantum yield, 0.89) for DY-505.

\section{In Vitro and In Vivo Whole-Body Spectral Imaging}

In vitro and vivo whole-body spectral imaging was performed using the whole-body optical NIRF scanner Maestro (Cambridge Research and Instrumentation, Inc.). For detection of DY-682 and DY-782 fluorescence signals, Maestro Excitation Filter Red (615-665 nm and >700-nm long pass) was used. To depict DY-505 and DY-782* signals, Maestro Excitation Filter Blue (445-490 nm) and Maestro Emission Filter Red (>700-nm long pass) were used. The acquired composite spectral data were unmixed into the spectrum of the respective fluorophores (20). For semiquantitative analysis of probe fluorescence signatures, regions of interest (2,000-2,500 pixels) were determined using the spectrally unmixed image of the respective optical probe. Fluorescence signatures were calculated from averaged counts, each representing the area under the curve of the respective unmixed probe spectrum. Average counts were normalized to pixel number and exposure time.

\section{In Vitro Imaging of Immobilized Probes on Protein Adsorbing (Western Blotting) Membranes}

Non- and IgG-conjugated dyes were spotted onto Western blotting membranes (e.g., nitrocellulose; Amersham Biosciences) in amounts of up to $0.2 \mathrm{nmol}$ for each dye (concentrations of up to $130 \mathrm{pmol} / \mathrm{cm}^{2}$ for each dye, in spots containing donor and acceptor molecules). The dried membrane was imaged with the wholebody NIRF scanner. The images were processed and analyzed as described for whole-body imaging. To determine the optimal relationship between donor and acceptor molecules (IgG-conjugated probe components), different donor-to-acceptor amounts were studied. In previous experiments, a donor-to-acceptor stoichiometry of 1 to 1 was determined to be optimal for the occurrence of FRET. Average donor-to-acceptor distances were estimated on the basis of the calculated dye concentration per spotted area, assuming a homogeneous distribution of the dyes.

\section{Cell Culture}

J774 murine macrophages (DSMZ) were grown in Dulbecco modified Eagle medium (Invitrogen) containing fetal calf serum (Gibco BRL, $10 \%[\mathrm{v} / \mathrm{v}]$ ) at $37^{\circ} \mathrm{C}$ in a $5 \% \mathrm{CO}_{2}$ atmosphere. 


\section{In Vitro Imaging of Stained Macrophages}

After cultivation of $5.5 \times 10^{5} \mathrm{~J} 774$ macrophages for $24 \mathrm{~h}$, the probes or free dyes were added $(5 \mathrm{~mL}$, final dye concentration of $1.2 \mathrm{nmol} / \mathrm{mL}$ ). The cells were cultured for a further $24 \mathrm{~h}$ and were then harvested by scraping and washed 3 times with phosphatebuffered saline. After centrifugation, the pellet was dissolved in $200 \mu \mathrm{L}$ of cell culture medium and pictured with the Maestro. To verify dye uptake, cells were analyzed microscopically via confocal laser scanning microscopy.

\section{Animal Experiments}

All procedures were approved by the regional animal committee and were in accordance with international guidelines on the ethical use of animals. Male Naval Medical Research Institute (NMRI) mice were housed under standard conditions with food and water ad libitum. Starting 7-14 d before the experiments, all mice received a low-pheophorbide diet (C 1039; Altromin) to reduce autofluorescence. At the beginning of the experiments, all animals were shaved at the area intended for imaging (head or back).

\section{In Vivo NIRF Imaging of Subcutaneously Injected Matrigel Cushions Bearing Probe-Stained Macrophages}

J774 macrophages $\left(1.5 \times 10^{7}\right)$ were incubated with a $1.2 \mathrm{nmol} / \mathrm{mL}$ concentration of IgG-DY-682 and IgG-DY-782 alone or in combination or remained unstained. Cell pellets were taken up in $100 \mu \mathrm{L}$ of Matrigel (BD Bioscience) and injected subcutaneously on the back of a mouse, so that each mouse bore 4 Matrigel cushions. For controls, mice received the same treatment but with the IgG-DY-682 replaced by IgG-DY-505. Both the IgG-DY-682-IgG-DY-782 and the IgG-DY-505-IgG-DY-782 groups consisted of 3 animals. The mice were imaged, and the acquired spectral imaging data were analyzed.

\section{In Vivo NIRF Imaging of Ear Edema in Mouse Model}

Ear edema was induced in accordance with a protocol from Kurnatowska and Pawlikowski (21). Twenty-eight male NMRI mice were divided into 7 groups consisting of 4 animals each. The different groups received the following treatments: untreated (group 1), only zymosan A injected (group 2), zymosan A injected and treated with IgG-DY-505 (group 3), IgG-DY-682 (group 4), IgG-DY-782 (group 5), IgG-DY-505 and IgG-DY-782 (group 6), or IgG-DY-682 and IgG-DY-782 (group 7). Edema was induced in all mice except the untreated native control group by injecting $20 \mu \mathrm{L}$ of a $1 \%(\mathrm{w} / \mathrm{v})$ suspension of zymosan A (Sigma-Aldrich $\mathrm{GmbH}$ ) into the root of the left ear. Immediately afterward, equimolar amounts of probes were intravenously injected into the tail veins of the mice of corresponding groups (amount corresponding to 55 nmol of dye per kilogram of body weight). The mice were imaged, and the acquired spectral imaging data were analyzed. To verify dye uptake in target cells, the mice were euthanized and cells from the edematous region were isolated by washing with phosphatebuffered saline. Subsequently, mononuclear cells were isolated via Histopaque1077 (Sigma-Aldrich) centrifugation (pooled isolated cells of each animal group). Isolated cells were attached onto poly-L-lysine-coated slices, fixed with $4 \%$ (w/v) formalin, washed with phosphate-buffered saline, stained with MOMA-2 (a rat monoclonal antibody to the monocyte and macrophage marker; dilution, 1:25; 60 min; room temperature) and Alexa488 (an antirat monoclonal antibody; dilution, 1:200; $60 \mathrm{~min}$; room temperature), and finally counterstained with Hoechst 33258 dye $(0.2 \mu \mathrm{g} / \mathrm{mL}$, $\left.10 \mathrm{~min}, 4^{\circ} \mathrm{C}\right)$. The cells were then imaged and analyzed via a confocal laser scanning microscope equipped with a laser diode (405 nm), an argon laser (458, 477, 488, and $514 \mathrm{~nm}$ ), and 2 helium-neon lasers (543 and $633 \mathrm{~nm}$ ).

\section{RESULTS}

\section{What Is the Constitution of the FRET Probe?}

The chosen dyes DY-682 (donor; absorption [abs]/ emission [em], 674/712 nm) and DY-782 (acceptor; abs/ em, 752/795 nm) display a significant spectral overlap between the donor emission and the acceptor absorption spectrum (Supplemental Fig. 1; supplemental materials are available online only at http://jnm.snmjournals.org) enabling efficient energy transfer. As a negative control, DY-505 (abs/em, 498/529 nm), displaying a fluorescence spectrum that has only little overlap with the absorption of DY-782, was used as the donor. This resulted in a low efficiency for energy transfer to DY-782. The chemical structures, spectroscopic properties, and FRET parameters of these fluorophores are summarized in Supplemental Figure 2 and in Table 1. After coupling the dyes to polyclonal rabbit $\operatorname{IgG}$ molecules, a dye-to-protein ratio of between 2.9 and 3.1 was achieved.

\section{Does FRET Occur When Probe Mixtures Are Adsorbed onto Western Blotting Membranes?}

The results from fluorescence measurements with the probes DY-505, DY-682, and DY-782 alone or in combination, spotted onto adsorbing membranes $(0.2 \mathrm{nmol}$ of each dye, corresponding to $130 \mathrm{pmol} / \mathrm{cm}^{2}$ ), are summarized in Figure 1. This model system was used to provide a dye concentration that is sufficiently high to result in small dye-to-dye distances, thereby favoring FRET. The fluorescence intensity of the donor DY-682 strongly decreases in the presence of the acceptor DY-782, and simultaneously, the emission of the latter is enhanced when DY-682 is added (Fig. 1A, left). For the control pair, no distinct influence of the presence of DY-505 on the signal derived from DY-782 could be detected, and vice versa (Fig. 1A, right). This finding was confirmed by semiquantitative analysis, in which each fluorescence signal represents the area under the curve of the respective unmixed spectrum. The emission of DY-782 is enhanced in the presence of DY-682, and simultaneously, the signal of DY-682 is diminished. The intensities of DY-505 and DY-782 are hardly affected when combined. Moreover, when the amounts of donor and acceptor are varied (constant donor-to-acceptor stoichiometry of 1 to 1), a distinct increase in acceptor fluorescence in the presence of the donor was observed at the highest amounts tested $\left(0.2 \mathrm{nmol}\right.$, corresponding to $130 \mathrm{pmol} / \mathrm{cm}^{2}$ for each dye, Figs. 1B and 1C), with a concomitant quenching of donor fluorescence. With a Förster radius of approximately $6 \mathrm{~nm}$ for DY-682, optimal donor-to-acceptor distances to result in FRET were achieved at amounts of $0.2 \mathrm{nmol}\left(130 \mathrm{pmol} / \mathrm{cm}^{2}\right.$ for each dye, estimated donor-toacceptor distance of $4 \mathrm{~nm}$ ). In contrast, this was not the case in relation to the DY-505-DY-782 couple. Given the 


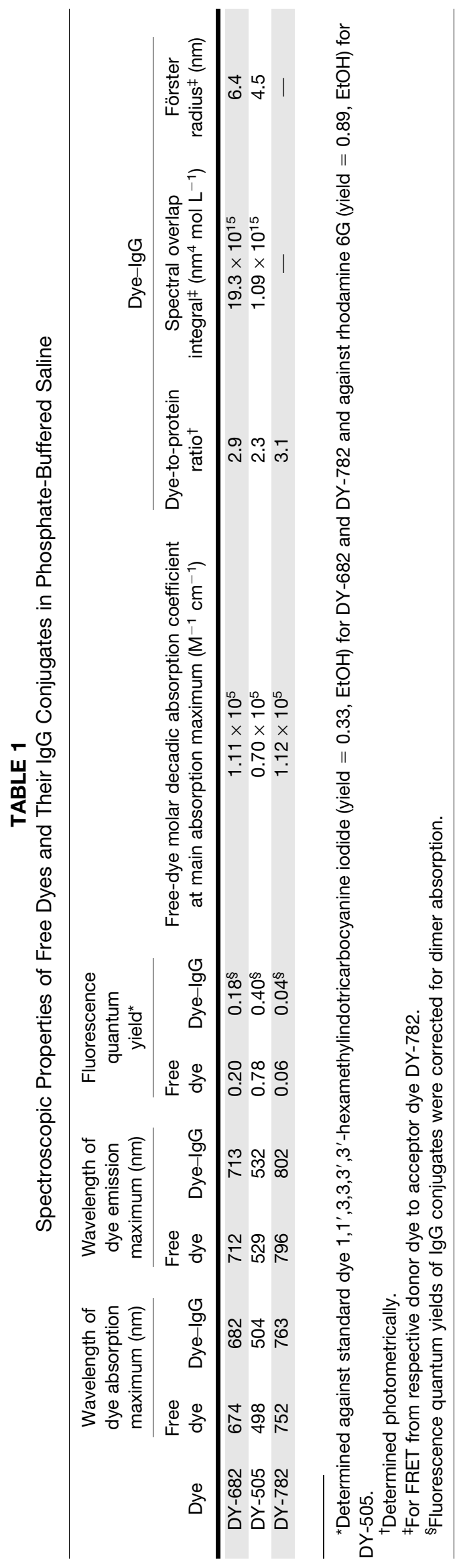

smaller Förster radius of DY-505 (4.5 nm), the dependency of donor-to-acceptor distances on concentration was by far less pronounced. By use of the IgG probes IgG-682 and IgGDY-782 (Fig. 1D), FRET was also detectable but not for the control pair (IgG-DY-505 and IgG-DY-782). Interestingly, a distinct dependency on probe concentration and, concomitantly, on donor-to-acceptor distance was observed (Fig. 1D) even after coupling of dyes to IgG molecules. These results corroborate the occurrence of FRET in vitro for the nearinfrared probe pair IgG-DY-682 and IgG-DY-782.

\section{Can FRET Be Detected When IgG-DY-682 and IgG-DY-782 Probes Are Packed into Phagocytotic Compartments of Macrophages?}

In a second step toward in vivo FRET imaging, we evaluated our IgG-conjugated dyes in vitro in cultivated J774 macrophage cells. The expected signaling of each of the donor and acceptor fluorophores alone or in the presence of its counterpart dye was observed after accumulation in endocytic and phagocytic cell compartments (Fig. 2). Semiquantitative analysis of cell pellets incubated with both IgGDY-682 and IgG-DY-782 revealed a distinct decrease in DY-682 fluorescence compared with that of IgG-DY-682 alone and an increase in the IgG-DY-782 signal. For the IgG-DY505-IgG-DY-782 system, only a slight change in IgG-DY505 fluorescence intensity and no change in IgG-DY-782 emission were observed. Comparable results were obtained when macrophages were analyzed after incubation with the native fluorophores of the FRET and control pair. These results suggest the occurrence of FRET in living biologic systems such as macrophages for the IgG-DY-682-IgG-DY782 system. The close proximity of both probes in target cells was shown by confocal laser scanning microscopy of macrophages that had been incubated with the probes, unveiling internalization into cytoplasmatic compartments (supplemental data).

\section{Does the Presence of Skin and Tissue Autofluorescence Affect Detectability of FRET In Vivo?}

For the envisioned exploitation of FRET in vivo for the simultaneous imaging of multiple probe-target interactions, it is essential that these effects are detectable through the skin and are not superimposed or falsified by tissue autofluorescence. Therefore, as a next step, we embedded labeled macrophages (unstained macrophages, after incubation with donor probe or acceptor probe alone or with donor and acceptor probe) and injected them subcutaneously into the back of NMRI mice. As follows from Figure 3 and as observed in previous in vitro experiments, the signal of IgGDY-682 is reduced in the presence of IgG-DY-782, and the fluorescence of IgG-DY-687 is enhanced for these locally accumulated cells in vivo. In the case of the IgG-DY-505IgG-DY-782 pair, acceptor IgG-DY-782 fluorescence (caused by direct acceptor excitation) showed no distinct change in intensity regardless of the presence of DY-505 (Fig. 3, 


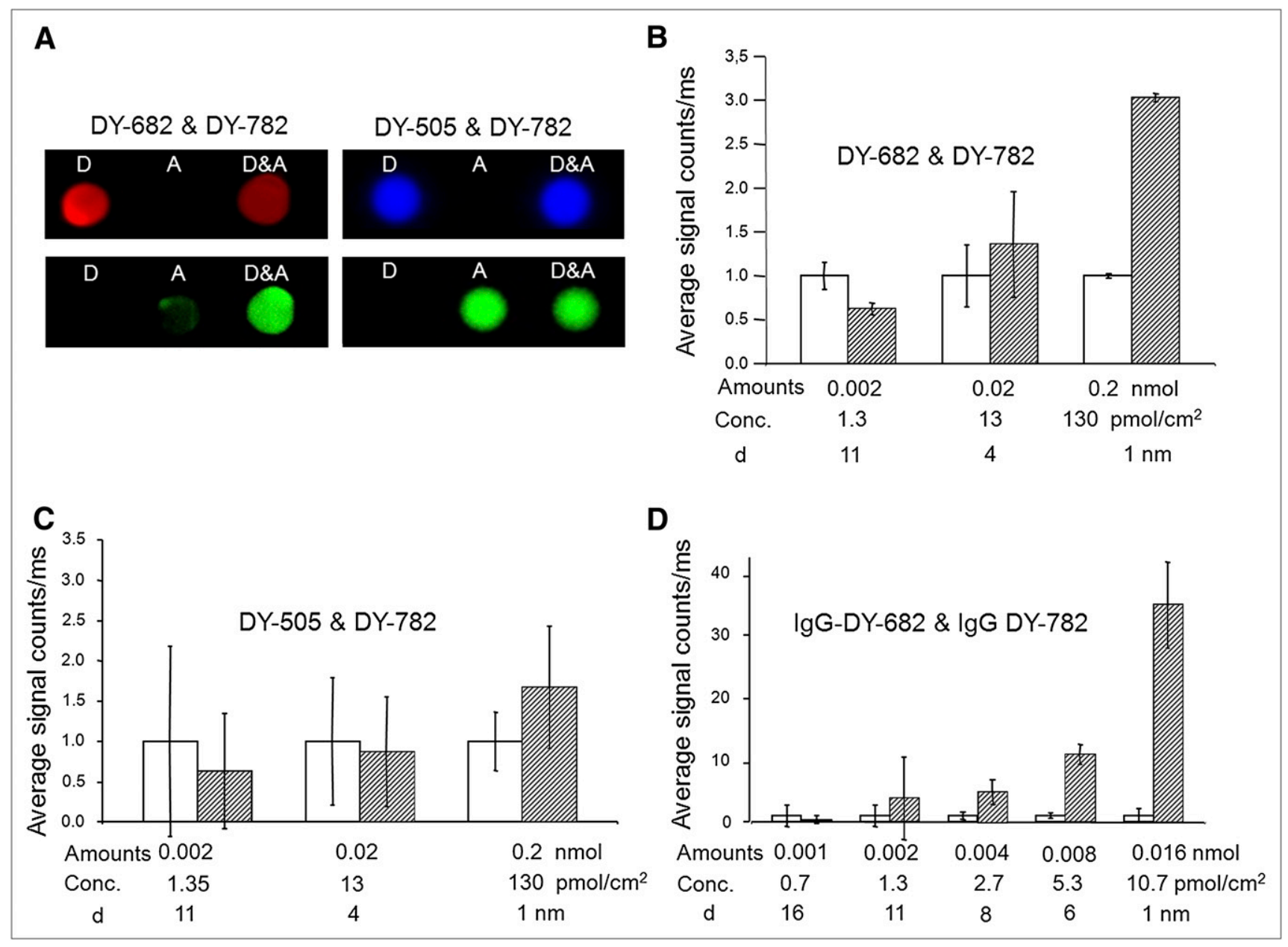

FIGURE 1. Acceptor fluorescence shows FRET behavior at defined concentrations of donors vs. acceptors. Defined concentrations (0.002-0.2 corresponding to $\left.1.3-130 \mathrm{pmol} / \mathrm{cm}^{2}\right)$ of DY-505, DY-682, DY-782 or combinations of DY-505 with DY-782 and DY-682 with DY-782 were spotted onto adsorbing membrane (1-to-1 stoichiometry), and fluorescence was imaged using whole-body NIRF imaging system. (A) Spectrally discriminated images of spots displaying signals derived from donor (DY-682: red, DY-505: blue) and acceptor probe (DY-782: green). (B and C) Semiquantitative analysis of acceptor fluorescence intensity alone (white bars) or in combination with donor (hatched bars). (D) Acceptor fluorescence of IgG-DY couples. $d=$ estimated donor-to-acceptor distance; conc = concentration of each dye per spot. Data are from 3 independent experiments.

bottom right). All data refer to a 30 -min postinoculation time. These results were confirmed by semiquantitative analysis of fluorescence intensities (Supplemental Fig. 3). Subsequent to inoculation of the labeled cells, the IgG-DY-682 signal alone was compared several times with the IgG-DY682 signal in the presence of DY-782 over a period of $24 \mathrm{~h}$. In each case, a reduced signal was observed. Simultaneously, the emission of IgG-DY-782 increased, with the highest intensity occurring $2 \mathrm{~h}$ after inoculation. For the control pair, the donor IgG-DY-505 was not distinctly quenched in the presence of the acceptor probe IgG-DY782. The IgG-DY-782 signal was initially high immediately after cell inoculation and remained almost unchanged in the presence or absence of the donor. Although the observed fluorescence intensities were generally lower than in the in vitro experiments, this in vivo model demonstrates that FRET could even be detected through the skin of living organisms.

\section{Does FRET Take Place After Systemic Probe Application in an In Vivo Mouse Ear Edema Model?}

FRET was observed to take place in vivo in an inflammation model: a zymosan A-induced ear edema model (Fig. 4) (21). After injection of the probes (55 nmol per kilogram of body weight) either separately or in combination into edematous mice, the IgG-DY-682 fluorescence strongly decreased in the presence of IgG-DY-782 (Fig. 4: DY-682 signal). Distinct IgG-DY-682-induced enhancement of the IgG-DY-782 emission (DY-782 signal) was observed up to $4 \mathrm{~h}$ after injection and became moderate after $6 \mathrm{~h}$ (Supplemental Fig. 4, semiquantitative analysis). In the case of the IgG-DY-505-IgG DY-782 system, the emission of IgG-DY-505 was reduced in the presence of IgG-DY-782 (Supplemental Figs. 4 and 5: DY-505 signal), similarly to the reduction in the cell inoculation model. However, the signal derived from IgG-DY-782 (Supplemental Figs. 4 and 5: DY-782* signal) remained unaffected. These results 


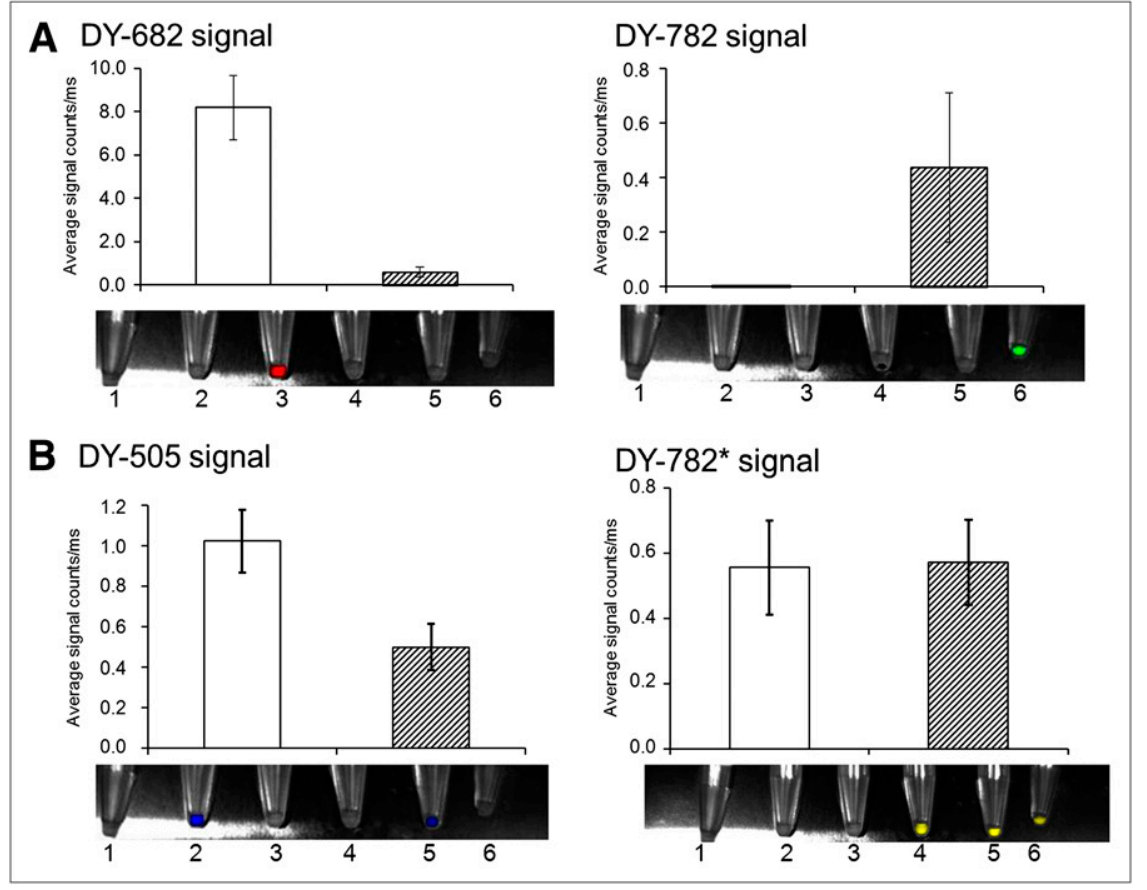

FIGURE 2. FRET effects can also be observed for DY-682 with DY-782 after internalization of dyes by murine macrophages J774. After incubation of $\mathbf{J 7 7 4}$ macrophages for $24 \mathrm{~h}$ with IgG-coupled dyes $(1.2 \mathrm{nmol} /$ $\mathrm{mL}$ ), cells were pelleted and imaged using whole-body NIRF imaging system. Semiquantitative analysis of fluorescence intensity was performed for each fluorophore alone (white bars) or in combination (hatched bars) (A: IgG-DY-682 with DY782; B: IgG-DY-505 with DY-782). Values are mean average of 3 different cell pellets and SEM. Bottom of each graph shows superimposed light and color-coded NIRF images of cell pellets after dye incubation. strongly suggest that FRET also occurs in vivo after systemic probe application. Analysis of isolated monocytes and macrophages in the area of the zymosan injection verified DY molecule uptake (Supplemental Fig. 6).

\section{DISCUSSION}

Nearly all pathophysiologic disease involves key activation processes represented by the interaction of two or more molecular species. In vivo image-based identification of such interactions should markedly foster the understanding of pathophysiologic processes, particularly in preclinical research activities. As revealed by our in vitro and in vivo spectral multiplexing approach, simple near-infrared targeted probes designed as a FRET pair are capable of cooperative signaling. This finding underlines the potential of this imaging strategy to unveil

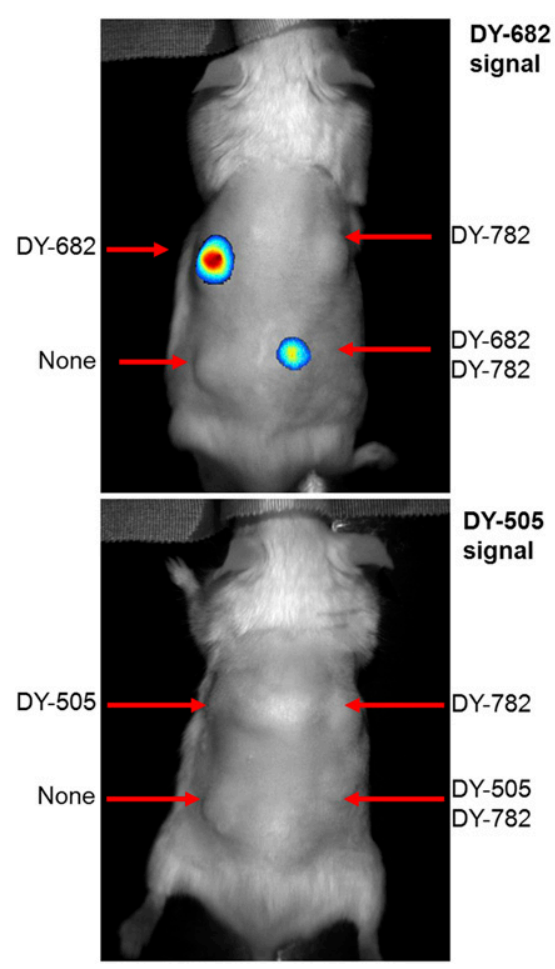

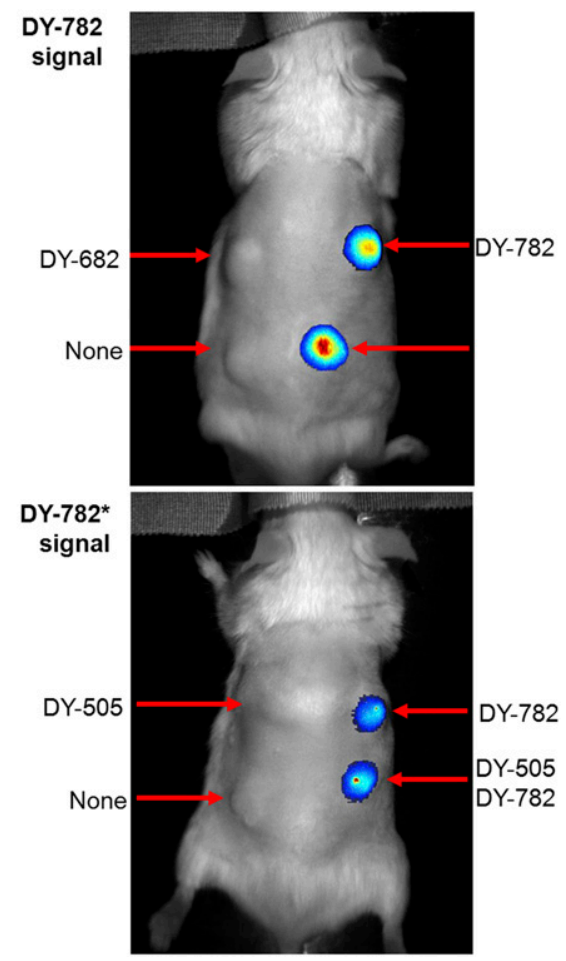

FIGURE 3. FRET effects can also be demonstrated after inoculation of labeled macrophages into mice. J774 macrophages were incubated with dye $(1.2 \mathrm{nmol} / \mathrm{mL})$-coupled IgG probes (each probe alone or in combination) for $24 \mathrm{~h}$ and injected subcutaneously on backs of mice. Representative visible and superimposed NIRF (false colors: blue [low fluorescence intensity] and red [high fluorescence intensity]) images of mice were obtained at $30 \mathrm{~min}$ after inoculation using whole-body NIRF imaging system. (Top) DY-682 and DY-782 signals of mouse injected with macrophages labeled with IgG-DY-682 and IgG-DY-782. (Bottom) DY505 and DY-782* signals corresponding to macrophages labeled with IgG-DY-505 and lgG-DY-782. 

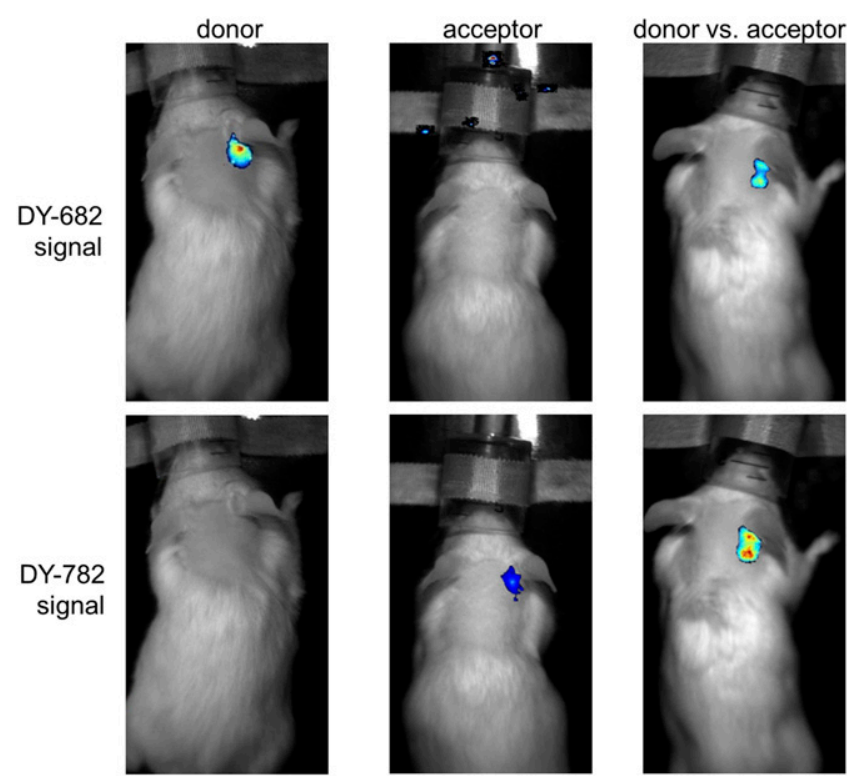

FIGURE 4. After systemic probe application of IgG-DY-682 vs. IgGDY-782, FRET effects are visible in mouse model of inflammatory ear edema. Representative images are shown of DY-682 and DY-782 signals of edematous mouse injected with IgG-DY-682 and IgG-DY782 ( $55 \mathrm{nmol}$ of dye per kilogram of body weight) $6 \mathrm{~h}$ after application using whole-body NIRF imaging system. Overlay is of fluorescence (false colors: blue [low fluorescence intensity] and red [high fluorescence intensity]) and white-light images. From left to right are shown localization of donor, acceptor, and donor in presence of acceptor.

the proximity of different biologic target structures of interest.

For the IgG-coupled fluorophores, the occurrence of FRET was detectable after direct application of the different probes spotted on a Western blotting membrane. In emission, spectral cross talk between donor and acceptor fluorescence can be excluded, as the emission profile of the acceptor (the IgG-DY-782) can be clearly discriminated from that of the donor via spectral analysis. The influence of the direct excitation of the acceptor was controlled by comparison of the fluorescence intensities obtained in the presence and absence of the donor. The influence of the distance between the donor and acceptor probe could be indirectly demonstrated by the fact that changes in FRET-related fluorescence were observed only at defined donor-acceptor concentrations (stoichiometry, 1 to 1 ). The fact that FRET was also observed after coupling of dye molecules to comparatively large IgGs is attributed to preferential dye binding to primary amino groups in the lysine-rich regions of the $\mathrm{Fc}$ domain of this molecule. Close proximity between donor and acceptor fluorophores was given by a distinct condensation of these $\mathrm{Fc}$ domains on Western blotting membranes and after probe internalization in endosomal compartments of phagocytes.

We showed that FRET can be detected when IgG-DY682-IgG-DY-782 pairs are packed into phagocytotic compartments of macrophages as a result of the phagocytotic activity of these cells. This brings the differently labeled probes into close proximity as a result of intracytoplasmic condensation into endolysosomes/phagosomes (22). The observed decrease of DY-682 fluorescence (compared with the respective chromophore alone) is a strong indication for energy transfer, thereby paralleling the results observed for probe mixtures on nitrocellulose membranes. The used chromophores are stable in the presence of a low $\mathrm{pH}$ and proteases (23), suggesting they should survive in an acidic and enzymatic milieu of endolysosomes after cell internalization.

In a following step, when the probes were systemically applied in a mouse edema model, FRET was detectable as well. Here, the necessary close proximity between the dyes to enable FRET signaling in vivo is ensured using an inflammatory model, where phagocytes are recruited $(24,25)$ and lead to an internalization of the antibody-based probes via Fc receptors. Dye internalization could be confirmed by microscopic analysis.

Interestingly, after systemic probe application, distinct probe accumulation kinetics at the edematous region were found, as derived from a comparison of time-dependent signaling in animals carrying implanted (labeled) macrophages with macrophages of edematous regions. The continuous decrease in signaling of implanted macrophages was probably caused by continuous cell division (population doubling time of macrophage cell line, $\sim 30 \mathrm{~h}$ [our own unpublished data]); migration of macrophages out of the edematous region (26); immune reactions against syngeneic but not allogenic cells, leading to their elimination; or a combination of these effects. In contrast, signaling after systemic probe application is mainly the result of continuous infiltration of the edematous region by phagocytes (27), which were previously labeled as a consequence of the intravenous application of the conjugated antibodies (28). It has been shown (29) that phagocytes are recruited from a splenic reservoir at the site of inflammation, implying that, in relation to the model used here, probes would be internalized by phagocytes on their way through circulation rather than at the inflammatory region itself.

Using the edema model, we have shown, for what is to our knowledge the first time, that FRET is possible when a contrast agent cocktail is administered into a nonengineered living organism. This is by far a simpler strategy than using systems in which chromophore-modified (fused) proteins are to be incorporated into cells in order to detect specific molecule interactions (7-9,30,31). Even though this in vivo approach represents a proof of concept, labeling of macrophages via FRET is expected to work also in other disease models related to spontaneous inflammatory processes, such as rheumatoid arthritis. Specific cell internalization processes may be elucidated in future. Even though potential clinical applications remain to be elucidated, the use of near-infrared chromophores is advantageous because of good tissue penetration and improved signal-to-noise ratios $(2,3)$, compared with approaches using luminescent or fluorescent proteins at shorter wavelengths. Accordingly, 
our data show that the occurrence of FRET is not falsified by tissue autofluorescence and is detectable through the skin.

One important aspect for in vivo intramolecular FRET, as shown here, is the need to apply donor and acceptor molecules with similar pharmacokinetics, allowing a reproducible stoichiometry between donor and acceptor probes at the target region. Here, this requirement was fulfilled, since the same antibody was used although with 2 different chromophores. Our proof of concept addressed also the functionality of probe internalization via macrophages. However, when more site-specific protein interactions are being addressed, the necessity to adjust the chemical constitution of the donor and acceptor molecules to the respective target might lead to varying pharmacokinetics and, consequently, to different stoichiometries. The result could be unfavorable conditions (e.g., in terms of donor-acceptor stoichiometry) under which FRET might be difficult to detect. Further challenges could arise in obtaining sufficient expression of target molecules and good accessibility to high-affinity probes so as to achieve reliable signaling in vivo.

The use of a whole-body multispectral imaging system in conjunction with spectral unmixing comprises a comparatively simple methodology for multiplexed biomarker analysis. In this context, the omission of a control system with the application of donor or acceptor probes per se would be advantageous. In contrast, the elucidation of structural components near each other cannot be obtained using conventional colocalization analysis of spectral signatures and superimposition of false color images, methods that have so far been used for in vivo imaging.

\section{CONCLUSION}

Our system-because it can be extended beyond the cellular processes shown here to molecular interactions via FRET signaling using target-specific probes-paves the way for new preclinical in vivo molecular imaging approaches. With respect to feasibility and gained advantages, in vivo near-infrared multiplexed imaging via FRET shows potential to become a new and robust tool for drug discovery and basic research in biomedicine.

\section{DISCLOSURE STATEMENT}

The costs of publication of this article were defrayed in part by the payment of page charges. Therefore, and solely to indicate this fact, this article is hereby marked "advertisement" in accordance with 18 USC section 1734.

\section{ACKNOWLEDGMENTS}

These investigations were supported by the German Research Foundation (DFG) within project HI 689/6-1 and by the Federal Ministry of Economics and Technology (BMWI-13/09). We gratefully acknowledge the experimentation and data analysis of Toni Luge, figure preparation by Susanne Kossatz and Jana Hölzer, and technical support by
Doreen May. No other potential conflict of interest relevant to this article was reported.

\section{REFERENCES}

1. Licha K. Contrast agents for optical imaging. Top Curr Chem. 2002;222:1-29.

2. Ntziachristos V, Ripoll J, Wang LHV, Weissleder R. Looking and listening to light: the evolution of whole-body photonic imaging. Nat Biotechnol. 2005;23: 313-320.

3. Resch-Genger U, Grabolle M, Cavaliere-Jaricot S, Nitschke R, Nann T. Quantum dots versus organic dyes as fluorescent labels. Nat Methods. 2008;5:763-775.

4. Sapsford KE, Berti L, Medintz IL. Materials for fluorescence resonance energy transfer analysis: beyond traditional donor-acceptor combinations. Angew Chem Int Ed Engl. 2006;45:4562-4589.

5. Fehr M, Frommer WB, Lalonde S. Visualization of maltose uptake in living yeast cells by fluorescent nanosensors. Proc Natl Acad Sci USA. 2002;99: 9846-9851.

6. Hoffmann C, Gaietta G, Bunemann M, et al. A FlAsH-based FRET approach to determine $\mathrm{G}$ protein-coupled receptor activation in living cells. Nat Methods. 2005;2:171-176.

7. John S, Weiss JN, Ribalet B. Subcellular localization of hexokinases I and II directs the metabolic fate of glucose. PLOS ONE. 2011;6:e.17674.

8. Veetil JV, Jin S, Ye KM. A glucose sensor protein for continuous glucose monitoring. Biosens Bioelectron. 2010;26:1650-1655.

9. Rusanov AL, Ivashina TY, Vinokurov LM, et al. Lifetime imaging of FRET between red fluorescent proteins. J Biophotonics. 2010;3:774-783.

10. Delagrave S, Hawtin RE, Silva CM, Yang MM, Youvan DC. Red-shifted excitation mutants of the green fluorescent protein. Biotechnology (N Y). 1995;13: $151-154$.

11. Tsien RY. The green fluorescent protein. Annu Rev Biochem. 1998;67:509-544.

12. Deissler V, Ruger R, Frank W, Fahr A, Kaiser WA, Hilger I. Fluorescent liposomes as contrast agents for in vivo optical imaging of edemas in mice. Small. 2008;4:1240-1246.

13. Lisy M-R, Goermar A, Thomas C, et al. In vivo near-infrared fluorescence imaging of carcinoembryonic antigen-expressing tumor cells in mice. Radiology. 2008;247:779-787.

14. Pauli J, Vag T, Haag R, et al. An in vitro characterization study of new near infrared dyes for molecular imaging. Eur J Med Chem. 2009;44:3496-3503.

15. Pauli J, Brehm R, Spieles M, Kaiser W, Hilger I, Resch-Genger U. Novel fluorophores as building blocks for optical probes for in vivo near infrared fluorescence (NIRF) imaging. J Fluoresc. 2010;20:681-693.

16. Matveeva EG, Terpetschnig EA, Stevens M, et al. Near-infrared squaraine dyes for fluorescence enhanced surface assay. Dyes Pigm. 2009;80:41-46.

17. Mujumdar SR, Mujumdar RB, Grant CM, Waggoner AS. Cyanine-labeling reagents: sulfobenzindocyanine succinimidyl esters. Bioconjug Chem. 1996;7: 356-362.

18. Resch-Genger U, Pfeifer D, Monte C, et al. Traceability in fluorometry: Part II. Spectral fluorescence standards. J Fluoresc. 2005;15:315-336.

19. Würth C, Grabolle M, Pauli J, Spieles M, Resch-Genger U. Comparison of methods and achievable uncertainties for the relative and absolute measurement of photoluminescence quantum yields. Anal Chem. 2011;83:34313439 .

20. Mansfield JR, Hoyt C, Levenson RM. Visualization of microscopy-based spectral imaging data from multi-label tissue sections. In: Current Protocols in Molecular Biology. Somerset, NJ: Wiley; October 2008: chapter 14, unit 14 19

21. Kurnatowska I, Pawlikowski M. Anti-inflammatory effects of somatostatin analogs on zymosan-induced earlobe inflammation in mice: comparison with dexamethasone and ketoprofen. Neuroimmunomodulation. 2001;9:119-124.

22. Stuart LM, Ezekowitz RAB. Phagocytosis: elegant complexity. Immunity. 2005;22:539-550.

23. Czerney P, Hartmann H. Houben-Weil, eds. Methoden der organischen Chemie. 4th ed. New York, NY: Georg Thieme Verlag Stuttgart; 1991.

24. Cohen J. The immunopathogenesis of sepsis. Nature. 2002;420:885-891.

25. Libby P, Ridker PM, Maseri A. Inflammation and atherosclerosis. Circulation. 2002;105:1135-1143.

26. Eisenblätter M, Ehrchen J, Varga G, et al. In vivo optical imaging of cellular inflammatory response in granuloma formation using fluorescence-labeled macrophages. J Nucl Med. 2009;50:1676-1682.

27. Ajuebor MN, Das AM, Virag L, Szabo C, Perretti M. Regulation of macrophage inflammatory protein-1 alpha expression and function by endogenous interleukin-10 in a model of acute inflammation. Biochem Biophys Res Commun. 1999;255:279-282. 
28. Ballou B, Fisher GW, Waggoner AS, et al. Tumor labeling in vivo using cyanineconjugated monoclonal antibodies. Cancer Immunol Immunother. 1995;41:257263.

29. Swirski FK, Nahrendorf M, Etzrodt M, et al. Identification of splenic reservoir monocytes and their deployment to inflammatory sites. Science. 2009;325:612616.
30. Arslanbaeva LR, Zherdeva VV, Ivashina TV, Vinokurov LM, Rusanov AL, Savitsky AP. Genetically encoded FRET-pair on the basis of terbium-binding peptide and red fluorescent protein. Appl Biochem Microbiol. 2010;46:154-158.

31. Rusanov AL, Savitsky AP. Fluorescence resonance energy transfer between fluorescent proteins as powerful toolkits for in vivo studies. Laser Phys Lett. 2011;8:91-102.

\section{Errata}

In the article, "MIRD Dose Estimate Report No. 20: Radiation Absorbed-Dose Estimates for ${ }^{111}$ In and ${ }^{90}$ Y-Ibritumomab Tiuxetan," by Fisher et al. (2009;50:644-652), Eq. 3 should have been $y=a e^{-b x}+c^{-d x}$. The results of this study are not affected by this correction. The authors regret the error.

In the article, "Preparation and Characterization of L- $\left[5-{ }^{11} \mathrm{C}\right]$-Glutamine for Metabolic Imaging of Tumors," by Qu et al. (2012;53:98-105), the name of the eighth author listed in the byline was incorrect. The correct name is Lewis A. Chodosh. The authors regret the error. 